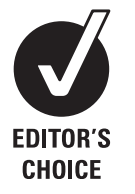

Division of Emergency Medicine, University of Cape Town, Cape Town, South Africa

Correspondence to Dr Heike Geduld, METRO EMS, Bellville 7535, South Africa; hgeduld@pgwc.gov.za

NETCARE Hospital Group and EMSSA (the Emergency Medicine Society of South Africal.

Accepted 16 July 2010 Published Online First 2 September 2010

\title{
Taxi driver training in Madagascar: the first step in developing a functioning prehospital emergency care system
}

\author{
Heike Geduld, Lee Wallis
}

\begin{abstract}
Background Prehospital care in developing countries is severely lacking. Few countries can afford the relatively expensive formalised Western model of a prehospital emergency medical system. The WHO has highlighted the development of layperson first responder programmes as the most basic step in the development of a functioning prehospital system.
\end{abstract}

Aim To describe the first training programme of its kind, run in Mahajanga, Madagascar. The faculty was invited by Mahajanga Medical School.

Methods and Results Local input was taken into account in developing the curriculum. 26 taxi drivers were invited to attend in cooperation with the local municipality. The faculty consisted of five instructors from the Division of Emergency Medicine and EMSSA, plus local doctors from University Hospital Mahajanga. The 1-day course included workshops on prehospital scene management, bleeding and broken bones, immobilisation and patient movement, and labour and delivery. The workshops made use of commonly available items only including packets, string and towels; French and Malagasy translators were available throughout.

Conclusions Both faculty and candidates deemed the course a success and plans for formal evaluation of knowledge and skill retention are underway. Future plans are to continue the training using local instructors and in rural districts.

Prehospital care in developing countries is severely lacking. ${ }^{1}$ In Africa there are very few countries with robust prehospital services. Given the large emergency care burden within Africa, the role of transferring people to hospitals often falls to bus, taxi and truck drivers. ${ }^{2-4}$ As previous studies have shown, the prehospital mortality rates are higher in these developing countries than in the developed world. ${ }^{5}$ It has been shown that a prehospital emergency care system saves lives but few countries can afford the relatively expensive formalised Western model. ${ }^{5}$ The WHO has highlighted prehospital care (particularly trauma care) as a priority and has issued guidelines on the development of prehospital care systems. ${ }^{6}$ Inherent in the guidelines is the development of layperson First Responder programmes as the most basic step in the development of a functioning prehospital system. ${ }^{6}$ This paper describes the first training programme of its kind, run in Mahajanga, Madagascar.

Madagascar is an island nation of 19 million people, situated in the Indian Ocean off the east coast of Mozambique. Best known for the export of Vanilla, Madagascar is ranked 210th in terms of GNP per capita. ${ }^{7}$ The World Bank Development Indicators estimate the malnutrition rate at 36\% for children under 5 years, and the under- 5 mortality rate is 106 per $1000 .^{7}$ The average life expectancy at birth is 57 years for men and 61 years for women. ${ }^{8}$ Although the country has two medical schools (in Antanarivo and Mahajanga), there are still only three doctors per 10000 population and $<1$ healthcare worker per 10000 population. ${ }^{8}$ Along with infectious diseases such as tuberculosis and malaria, road traffic deaths account for a significant part of the mortality ( 5 per 1000 deaths). ${ }^{8}$ There is no formal prehospital medical system on the island and the large distances and rugged terrain hamper access to the central hospitals.

The Malagasy culture and history has strong French and Asian links but academics are now looking to form ties with their Southern African neighbours. Such ties were recently formed for Emergency Medicine with the University of Cape Town. In January 2010, a contingent of emergency physicians and emergency nurses from the Emergency Medicine Society of South Africa (EMSSA) were invited by the Paediatric Faculty of Mahajanga University Medical School to teach a layperson First Responder course as part of the Emergency Medicine outreach programme.

\section{BACKGROUND}

Mahajanga is a city on the west coast of Madagascar. It has a population of around 150000 . The central university hospital is the referral centre for the district and the home of the medical school. The medical school trains undergraduates and has postgraduate training programmes in medicine, surgery, paediatrics, and obstetrics and gynaecology.

Within the borders of Mahajanga, there is no functioning ambulance system, although in the university hospital grounds a vehicle transports patients between wards and the radiology department. Patients arrive at the hospital via private transport, buses and taxis. An ambulance vehicle had been donated to the Red Cross Service on the island but financial and mechanical constraints had left this non-operational. It had been staffed by a first aider and a driver. Prior to the training, discussions were held with local stakeholders including the district health managers, the medical school, local doctors and nurses, and the mayor's office. Development of an effective prehospital 
emergency system was identified independently as a priority for the health service in the district.

\section{TRAINING PROGRAMME}

The model of first responder programmes for lay people has already been utilised and validated in developing countries around the world. ${ }^{2-4}$ We based our training programme on these models and, on request by the target group, incorporated aspects of maternal care and delivery: birth asphyxia accounts for $25 \%$ of neonatal deaths in Madagascar. ${ }^{8}$ On questioning, many of the taxi drivers described women delivering in their vehicle. On this basis it was decided to include aspects of basic birthing care into the programme.

As with other First Responder programmes, the more common aspects of scene management, first aid splinting, haemorrhage control and immobilisation were also covered. Emphasis was placed on universal precautions and simple spinal precautions for trauma patients.

The programme consisted of four workshops:

1. Prehospital scene management: The focus of this workshop was on aspects of scene safety, including command and crowd control. A discussion of local resources and referral patterns was included to make it locally appropriate.

2. Bleeding and broken bones: This workshop focused on the practical aspects of haemorrhage control and splinting of fractures. Emphasis was placed on using commonly available items such as safety pins and newspapers.

3. Immobilisation and patient movement: Given local resources and the level of training, the trainers decided to keep focus on patient transfer and patient movement as a whole, rather than only cervical spinal immobilisation.

4. Labour and delivery: This practical workshop focused on how to assist and position the woman in the vehicle. It emphasised how assistance could be rendered if requested.

The key components of the course were the incorporation of hands-on practical training as this has been proven to be the most effective way to ensure knowledge retention. ${ }^{9}$ The use of translators allowed first language understanding (French and Malagasy). The workshops only made use of commonly available items, for example blankets, towels, sticks and plastic bags. A packaged or commercial First Aid kit was not felt to be practical in this environment.

The faculty consisted of five trainers (doctors and nurses) from the Division of Emergency Medicine at the University of Cape Town and EMSSA. Local doctors from the University Hospital, Mahajanga were invited to be instructors to familiarise them with the material in order to develop continuity, and to provide locally appropriate input.

Equipment for teaching was locally sourced. The cost of catering and translators was borne by Mahajanga University.

The 1-day course was held in municipal offices in the centre of Mahajanga. Twenty-six taxi drivers attended. These drivers were selected by the local municipality. Participants were enthusiastic and engaged in the practical workshops. Pen and paper were provided and most candidates made their own notes during the workshops. A question-and-answer session was held at the end of the course, allowing participants to clarify concepts and give feedback to the faculty. At this time participants were encouraged to develop their own makeshift First Aid kit to keep in their taxis: these included everyday items such as plastic packets, safety pins, newspapers and towels. At the end of the day they received a certificate of completion.

\section{DISCUSSION}

The taxi drivers, and the local and international faculty all agreed that the course was successful. It has empowered the participants, given them some basic first-aid knowledge and created an awareness in the community of the need for prehospital emergency services. It remains to be seen how this workshop will impact on the community at large in terms of acceptance from the public and implementation of the skills by the taxi drivers. While the programme tried to remain sensitive to local culture and norms, many of the trainees were concerned about how the community would react to them using their newly gained skills.

The advantages of this First Responder programme are its low tech, low cost approach which can be rolled out in any environment. The involvement of local instructors provided both local context and translation of medical terms during the course. These instructors are now able to teach the course, developing a basis for continuity. The programme was designed to be locally appropriate, focusing on the issues central to healthcare in Mahajanga. Although the team had limited time with the participants, both faculty and candidates deemed it to be adequate. It was felt that it was more important to cover a few basic concepts well and allow for hands-on practice rather than attempt to teach a larger number of topics.

Already there are plans to repeat this course together with the local instructors, not just within the city but in the rural districts as well.

An important element in rolling out such a programme is an evaluation of its effectiveness by assessing the knowledge and skills of those trained. ${ }^{3} 10$ A formal follow-up is planned but informal communication with local staff has suggested a positive impact with anecdotal reports of patients transferred more confidently to hospital by taxi drivers.

While getting the existing ambulance functioning again is a priority, a First Responder training programme would be an important initial step in the development of a prehospital emergency care system in Madagascar. ${ }^{11}$ Even the most basic system can have positive effects on the health outcomes, particularly in vulnerable subgroups such as paediatric and obstetric patients. ${ }^{12}$

Acknowledgements The programme was developed in conjunction with the Mahajanga University Hospital. Special thanks are extended to NETCARE Hospital Group and EMSSA (the Emergency Medicine Society of South Africa), for financial support

\section{Competing interests None.}

Provenance and peer review Not commissioned; not externally peer reviewed.

\section{REFERENCES}

1. Mock C. Strengthening prehospital trauma care in the absence of formal emergency medical services. World J Surg 2009;33:2510-11.

2. Tiska MA, Adu-Ampofo M, Boayke G, et al. A model of prehospital trauma training for lay persons devised in Africa. Emerg Med J 2004;21:237-9.

3. Wisborg T, Murad MK, Edvardsen 0, et al. Prehospital trauma system in a lowincome country: system maturation and adaptation during 8 years. J Trauma 2008; 64:1342-8

4. Jayamaran S, Mabweijano J, Lipnick $M$, et al. First things first: effectiveness and scalability of a basic prehospital trauma care program for lay first-responders in Kampala, Uganda. PLoS One 2009;4:e6955.

5. Mock C, Quansah R, Krishnan R, et al. Strengthening the prevention and care of injuries worldwide. Lancet 2004;363:2172-9.

6. Sasser S, Varghese M, Kellerman A, et al. Prehospital trauma care systems. Geneva, Switzerland: World Health Organization, 2005.

7. The World Bank. World development indicators. 2010. http://data.worldbank.org/.

8. WHO Statistical Information System (WHOSIS), http://www.who.int/whosis (accessed 4 Jun 2010). 
9. Husum H, Gilbert M, Wisborg T. Training pre-hospital trauma care in low-income countries: the 'Village University' experience. Med Teach 2003;25:142-8.

10. Mock C, Tiska M, Adu-Ampofi M, et al. Improvements in prehospital trauma care in an African country with no formal emergency medical services. J Trauma 2002;53:90-7.
11. Kobusingye OC, Hyder AA, Bishai D, et al. Emergency Medical systems in low-and middle-income countries: recommendations for actions. Bull World Health Organ 2005;83:626-31.

12. Razzak J, Kellerman AL. Emergency Medical Care in developing countries: is it worthwhile? Bull World Health Organ 2002;80:900-5.

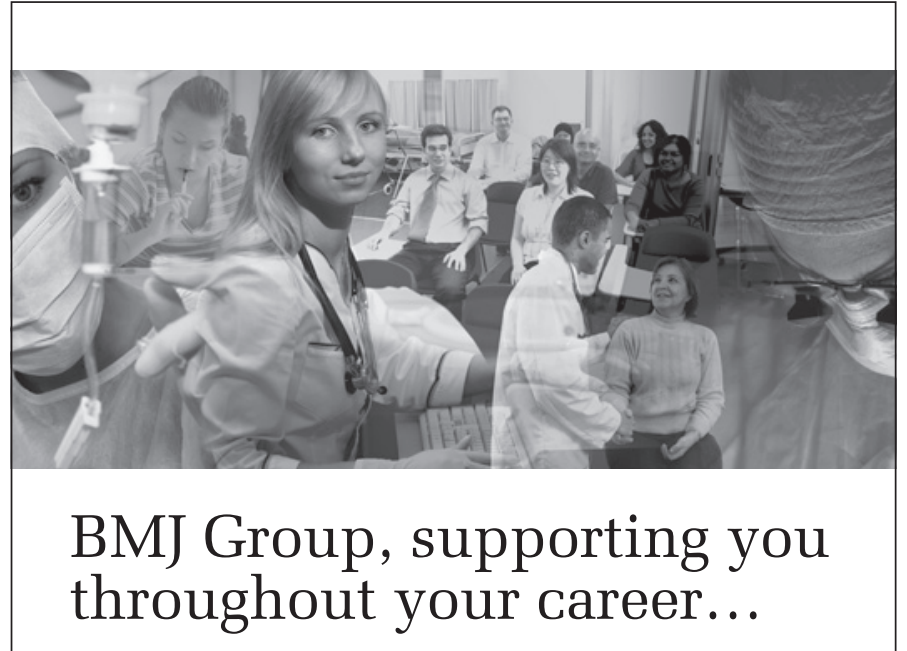

At BMJ Group we have resources available to you at every stage of your career.

Whether you are a medical student or doctor in training looking to keep up with the latest news and prepare for exams, or a qualified doctor who wants the latest medical information, to attend conferences, or looking for your next job, BMJ Group has something to offer. For the latest information on all of our products and services register to receive email updates at

group.bmj.com/registration

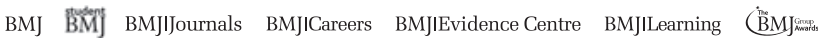

